\title{
A Study on the Coexistence of Fixed Satellite Service and Cellular Networks in a mmWave Scenario
}

\author{
Francesco Guidolin $^{\dagger *}$ Maziar Nekovee* Leonardo Badia ${ }^{\dagger} \quad$ Michele Zorzi ${ }^{\dagger}$ \\ $\dagger$ Dept. of Information Engineering, University of Padova, via Gradenigo 6/B, 35131 Padova, Italy \\ * Samsung R\&D Institute UK, Communications House, South Street, Staines-upon-Thames.Surrey TW18 4QE, UK
}

\begin{abstract}
The use of a larger bandwith in the millimeter wave (mmWave) spectrum is one of the key components of next generation cellular networks. Currently, part of this band is allocated on a co-primary basis to a number of other applications, such as the fixed satellite services (FSSs). In this paper, we investigate the coexistence between a cellular network and FSSs in a mmWave scenario. In light of the parameters recommended by the standard and the recent results presented in the literature on the mmWave channel model, we analyze different BSs deployments and different antenna configurations at the transmitters. Finally, we show how, exploiting the features of a mmWave scenario, the coexistence between cellular and satellite services is feasible and the interference at the FSS antenna can be kept below recommended levels.
\end{abstract}

Index Terms-Millimeter wave communication; cellular networks; radio spectrum management; satellite communication; beamforming.

\section{INTRODUCTION}

One of the primary contributors to global mobile traffic growth is the increasing number of wireless devices that are accessing mobile networks. Each year, several million new devices with different form factors and increased capabilities are being introduced. Over half a billion (526 million) mobile devices and connections were added in 2013 and the overall mobile data traffic is expected to grow to 15.9 exabytes per month by 2018, nearly an 11-fold increase over 2013 [1]. One of the most promising technologies to support the fast growing demand of high rate connectivity in next generation mobile cellular networks is the use of wide bands in millimeter wave (mmWave) frequency ranges. In particular, cellular allocations today are largely constrained under $6 \mathrm{GHz}$ but it will be possible to obtain a spectrum 200 times greater by exploiting the frequencies between 20 and $300 \mathrm{GHz}$.

Recent studies demonstrate the feasibility of mmWave mobile communications using multiple antenna arrays in conjunction with adaptive beamforming in order to compensate far propagation losses at high frequencies [2]. Some experimental results obtained using a prototype developed by Samsung are reported in [3]. The small wavelengths of these frequencies (in fact, of the order of $10^{-3} \mathrm{~m}$ ) allow to use large arrays of antennas to support directional beams to the users. A hybrid analog-digital beamforming scheme that exploits the mmWave channel and an antenna array with a low implementation complexity is presented in [4], and several measurements and capacity studies recently performed in New York City at 28 and $73 \mathrm{GHz}$ are presented in [5]. These studies are used in [6][7] to develop statistical channel models including pathloss, number of spatial clusters, angular dispersion and outage probability. In general, even in non-line-of-sight scenarios, strong signals can be detected 100 to $200 \mathrm{~m}$ from the base station (BS) and spatial multiplexing can be supported. Similar channel models based on indoor and outdoor measurements are presented in [8].

One of the candidate bands for the deployment of mmWave cellular mobile networks is the portion of spectrum between 17 and $30 \mathrm{GHz}$. Currently, part of this band is allocated on a co-primary basis to fixed services (FSs), cellular network backhaul, and fixed satellite services (FSSs) [9]. FSS is the official classification for geostationary communications satellites that provide, for instance, broadcast feeds to television stations, radio stations and broadcast networks. The FSS uplink (from FSS to satellite) is allocated in the band from 27.5 to $30 \mathrm{GHz}$ and the downlink (from satellite to FSS) is allocated from 17.3 to $21 \mathrm{GHz}$ [10]. Fig. 1 shows the spectrum allocations of the FSSs and high density FSSs (HDFSS) designated by the International Telecommunication Union (ITU) [10]. Therefore, it is important to study the possible coexistence between FSSs and mmWave cellular base stations (BSs) in order to preserve the functionalities of the satellite services.

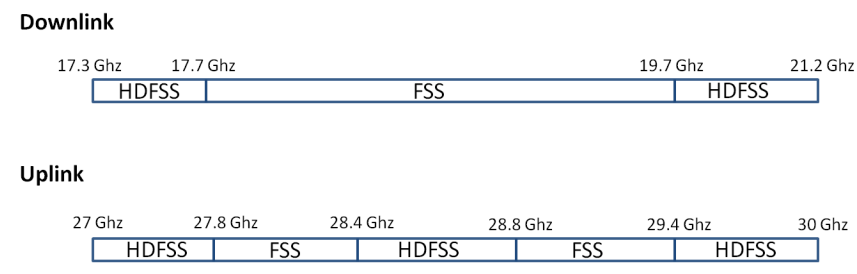

Fig. 1. FSSs and HDFSSs spectrum allocation

ITU investigated the spectrum sharing between FSSs and IMT-advanced systems in the frequency band from 3.4 to 6.4 $\mathrm{GHz}$ considering the devices as primary and secondary users respectively [11][12]. The aim of these works is to evaluate the interference at the FSS and to design possible interference mitigation techniques able to guarantee a minimum signal to interference ratio (SIR) at the primary user. Several techniques can be applied to mitigate the interference towards the FSS and improve the BS-FSS coexistence. Some cognitive Sat- 
Coms underlay, interweave and database related techniques to manage the BS-FSS interference are presented in [13]. An interference mitigation technique based on a nullsteering multi-user multiple-input-multiple-output (MU-MIMO) spatial division access scheme for frequency sharing between an IMTadvanced system and FSSs is proposed in [14]. A study on the separation distance between geostationary satellite communications (GSO) and terrestrial network in Ka band that guarantees a fixed interference to noise level $(\mathrm{I} / \mathrm{N})$ at the primary receiver is presented in [15]. A transmit beamforming technique at the BS to maximize the signal to interference plus noise ratio (SINR) towards the desired secondary user and to mitigate the interference towards the primary satellite terminals is proposed in [16].

Differently from this literature, in our work we study the coexistence between FSSs and mobile cellular BSs in the mmWave bands. In particular, we consider the parameters and the FSSs radiation pattern provided by the ITU and the channel models provided by the literature in these frequency bands. We analyze the level of $\mathrm{I} / \mathrm{N}$ at the FSS considering various multiple antenna configurations at the $\mathrm{BS}$ and different deployments of the mobile transmitters when no cooperation is allowed between the BSs. In particular, starting from the lower bound, represented by an omnidirectional configuration of the transmitters, we extend our work to the analysis of large antenna arrays that will be used in the new generation of mobile cellular systems. Finally, we show that exploiting a large number of antennas at the BSs and properly setting the protection distance between FSS and mobile BS, coexistence between the two systems is feasible.

The paper is organized as follows. Section II describes the model considered, focusing on the standard system parameters used in the analysis and Section III gives the performance evaluation for the different scenarios considered. Finally, conclusions and future works are drawn in Section IV.

Remark: Throughout the paper, we use boldface letters for vectors and matrices, and we denote with $(\cdot)^{T}$ the conjugate transpose.

\section{SySTEM MODEL}

In this paper we consider the frequency allocation at $18 \mathrm{GHz}$ that corresponds to the downlink band of the FSS system. The scenario is depicted in Fig. 2. We define as primary link the transmission from the satellite to the FSS while as secondary link the connection from a cellular BS to a mobile user equipment (UE) is considered. An additional interfering link is present from the BS to the FSS. A scheduling algorithm for this scenario has been proposed and studied in [17].

We can compute the interference (on a log scale) generated on such a link by BS $n$ to the FSS as

$$
I_{n}=P_{B S}+G_{F S S}(\phi)+G_{B S}-L(d)
$$

where $P_{B S}$ is the BS transmission power, $G_{F S S}(\phi)$ is the FSS antenna gain in the direction $\phi, G_{B S}$ is the BS antenna gain, $\phi$ is the angle between the main FSS antenna lobe and BS $n$, and $L(d)$ is the pathloss component at distance $d$, which in turn is the FSS-BS distance.

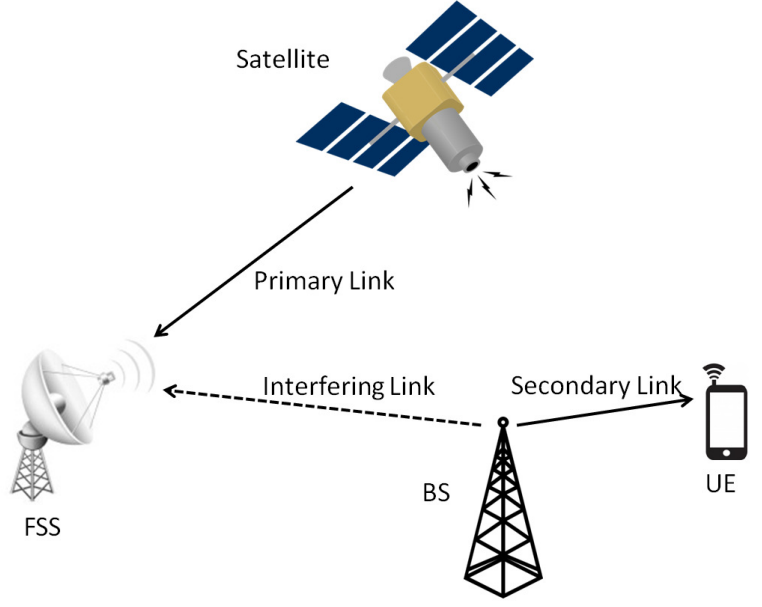

Fig. 2. FSS-BS coexistence scenario

The FSS antenna gain is computed as a function of the offboresight angles, which can be calculated using the model in [18]. Considering $\vartheta$ as the azimuth of the BS w.r.t. the FSS Rx main lobe, the off-boresight angle $\phi$ of the BS towards the FSS can be calculated as

$$
\phi=\arccos (\cos (\alpha) \cos (\epsilon) \cos (\vartheta)+\sin (\alpha) \sin (\epsilon))
$$

where $\alpha$ is the FSS elevation angle and $\epsilon$ is computed as:

$$
\epsilon=\frac{h_{t}-h_{s}}{d}-\frac{d}{2 r}
$$

where $h_{s}$ and $h_{t}$ are the heights of the BS and the FSS in meters, respectively, while $r$ is the effective Earth radius $(\approx$ $\left.8.5 \cdot 10^{6} \mathrm{~m}\right)$. The FSS off-boresight antenna gain pattern in $\mathrm{dB}$ can be computed as [19]:

$$
G_{F S S}(\phi)=\left\{\begin{array}{cl}
G_{\max } & \text { if } 0^{\circ}<\phi<1^{\circ} \\
32-25 \log \phi & \text { if } 1^{\circ} \leq \phi<48^{\circ} \\
-10 & \text { if } 48^{\circ} \leq \phi \leq 180^{\circ}
\end{array}\right.
$$

where $G_{\max }$ is the main beam axis FSS antenna gain.

Since mmWave channels are expected to have limited scattering, we adopt a double-directional geometry based stochastic model with $L$ scatterers [20]. Assuming $N_{U E}$ antennas at the UE and $N_{B S}$ antennas at the BS the $N_{U E} \times N_{B S}$ channel matrix $\mathbf{H}$ can be computed as

$$
\mathbf{H}=\sqrt{\frac{N_{B S} N_{U E}}{L}} \sum_{\ell=1}^{L} \alpha_{\ell} \mathbf{a}_{U E}\left(\gamma_{\ell}^{U E}\right) \mathbf{a}_{B S}^{*}\left(\gamma_{\ell}^{B S}\right)
$$

where $\alpha_{\ell}$ is the complex gain of the $\ell^{t h}$ path and $\gamma_{\ell}^{U E}, \gamma_{\ell}^{B S} \in$ $[0,2 \pi]$ are uniformly distributed random variables that represent the angles of arrival and departure, respectively. Finally, $\mathbf{a}_{U E}$ and $\mathbf{a}_{B S}$ are the antenna array responses at the UEs and $\mathrm{BSs}$ respectively. Assuming uniform linear arrays, $\mathbf{a}_{B S}$ can be written as

$\mathbf{a}_{B S}=\frac{1}{\sqrt{N_{B S}}}\left[1, e^{j \frac{2 \pi}{\lambda} D \sin \left(\gamma_{l}^{B S}\right)}, \ldots, e^{j\left(N_{B S}-1\right) \frac{2 \pi}{\lambda} D \sin \left(\gamma_{l}^{B S}\right)}\right]$ 
where $D$ is the distance between antennas. Similarly, $\mathbf{a}_{U E}$ can be computed by substituting $N_{B S}$ and $\gamma_{\ell}^{B S}$ in (5) with $N_{U E}$ and $\gamma_{\ell}^{U E}$, respectively.

We assume that the $\mathrm{BS}$ antenna gain in $\mathrm{dB}$ is

$$
G_{B S}=G_{o m n i}+G_{B F}
$$

where $G_{o m n i}$ is the conventional antenna gain when no beamforming techniques are applied and $G_{B F}$ is the beamforming gain. In this work, we adopt an RF beamforming where the gain is obtained by controlling phase and magnitude of the input signal to each antenna to form a directional beam in a particular direction. In order to reduce the system complexity, we assume that the BS can select the beam configuration within a predefined beam codebook with cardinality $N_{t}$ that uniformly covers the azimuth directions around the BS. In particular, the codebooks at the transmitter and the receiver are formed by $N_{t}$ and $N_{r}$ weight vectors $\left\{\mathbf{v}_{1}, \ldots, \mathbf{v}_{N_{t}}\right\}\left\{\mathbf{w}_{1}, \ldots, \mathbf{w}_{N_{r}}\right\}$ of sizes $N_{B S}$ and $N_{U E}$, respectively. Each vector is computed as $\mathbf{v}_{i}=\mathbf{a}\left(\rho_{i}\right)$ and $\mathbf{w}_{k}=\mathbf{a}\left(\theta_{k}\right)$ where $\rho_{i}$ and $\theta_{k}$ are the azimuth angles for the $i$-th transmit RF beam and $k$-th receive $\mathrm{RF}$ beam. We assume a multiple-input-single-output (MISO) scenario, in which the beamforming gain in the direction of the FSS antenna is:

$$
G_{B F}=10 \log \left(\left|\mathbf{v}_{i}^{T} \mathbf{h}_{F S S}\right|^{2}\right)
$$

where $\mathbf{v}_{i}$ is the beamforming precoding vector selected by the $\mathrm{BS}$ and $\mathbf{h}_{F S S}$ is the channel matrix between the BS and the FSS.

Recommendation [21] indicates that interference from fixed service systems should not cause the BER to exceed $10^{-4}$ for more than $0.03 \%$ of any month nor cause the BER to exceed $10^{-3}$ for $0.005 \%$ of any month. These interference allowances, in terms of percentage of system noise, can be converted into corresponding values of $\mathrm{I} / \mathrm{N}$. For this percentage of time, referred in the literature as "short term" interference, the corresponding $\mathrm{I} / \mathrm{N}$ values are equal to -2.4 and $0 \mathrm{~dB}$, respectively. To evaluate the level of interference at the FSS we consider a "long term" interference criterion that refers to a percentage of time greater than $20 \%$. In this case, recommendation [21] allows an interference level equivalent to $10 \%$ of the clear-sky satellite system noise that would give rise to a BER of $10^{-6}$. The recommended $\mathrm{I} / \mathrm{N}$ value is computed in [22] and is equal to $-10 \mathrm{~dB}$.

\section{A. Scenarios Considered}

We evaluate the interference at the FSS using three different scenarios.

In the first scenario we consider a single BS equipped with one omnidirectional antenna to evaluate the impact of the BS position and the elevation angle on the interference at the FSS. In this case, the BS antenna gain is given only by the omnidirectional component.

In the second scenario, we evaluate the aggregate interference from multiple omnidirectional BSs deployed in circular tiers around the FSS with fixed inter-site distance. The interference at the FSS is given by the sum of all the BS contributions that depend on the transmitter positions and on the FSS elevation angle. We define as protection distance $d_{p}$ the distance from the FSS and the first tier of BSs. The scenario is depicted in Fig. 3.

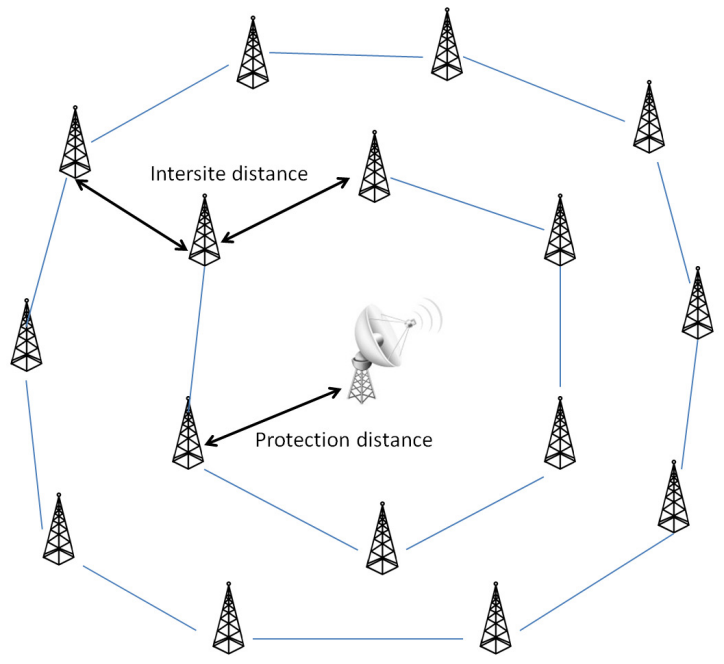

Fig. 3. Multiple BSs scenario

In the third scenario, we consider multiple directional BSs. In this case, the BSs, deployed as in the previous scenario, are equipped with multiple antennas and an RF beamforming scheme is adopted to serve the single antenna UEs. The users are randomly distributed within the BS coverage area and each BS selects one user at a time. The precoding beam vector is selected by each BS within the predefined beam codebook to maximize the SNR at the UE selected. We assume that the user scheduling and the beamforming selection are performed in a distributed manner among the BSs without any cooperation or signalling for interference coordination.

\section{PERFORMANCE EVALUATION}

In this section, we describe the results obtained for the scenarios presented in the previous section. We assume that the total downlink bandwith is $500 \mathrm{MHz}$ and the BSs allocate the power uniformly over this bandwith. The pathloss model is given exploiting the results presented in [8] on the mmWave band. Assuming a system effective noise temperature $\mathrm{T}$ equal to $300 \mathrm{~K}$, the one-sided noise power spectral density value results equal to $N_{0}=\mathrm{kT}=-143.82 \mathrm{dBW} / \mathrm{MHz}$, where $\mathrm{k}$ is the Boltzmann constant. The detailed system parameters are reported in Table I. All the evaluations have been performed using a customized MATLAB simulator.

\section{A. Single omnidirectional BS}

In Fig. 4, we evaluate the region around the FSS where a single omnidirectional BS can be placed without causing $\mathrm{I} / \mathrm{N}$ to go above the recommended threshold. In particular, the graph shows the contour of the " $-10 \mathrm{~dB}$ region" that represents the area where a single omnidirectional BS generates an amount of interference at the FSS higher than the regulatory requirements. We evaluate the regions obtained with a pico $\mathrm{BS}, P_{B S}=30$ $\mathrm{dBm}$ and with a macro $\mathrm{BS}, P_{B S}=43 \mathrm{dBm}$, considering the 
TABLE I. MAIN SYSTEM PARAMETERS

\begin{tabular}{|c|c|}
\hline \multicolumn{1}{|c||}{ Parameter } & Value \\
\hline Carrier frequency & $18 \mathrm{GHz}$ \\
\hline Total downlink bandwidth & $500 \mathrm{MHz}$ \\
\hline BS transmit power & $30,43 \mathrm{dBm}$ \\
\hline BS antenna height & $20 \mathrm{~m}$ \\
\hline BS omnidirectional antenna gain & $6,12 \mathrm{dBi}$ \\
\hline BSs intersite distance & $500 \mathrm{~m}$ \\
\hline BS inter-antenna distance & $\lambda / 2$ \\
\hline BS beam codebook cardinality & 16 \\
\hline FSS antenna main lobe gain & $42.1 \mathrm{dBi}$ \\
\hline FSS antenna diameter & $2.4 \mathrm{~m}$ \\
\hline FSS antenna height & $2 \mathrm{~m}$ \\
\hline Elevation angle & $10^{\circ}, 30^{\circ}$ \\
\hline Pathloss model & $31.39+10 \times 2.47 \log (d)[8]$ \\
\hline Number of scatterers & $300 \mathrm{~K}$ \\
\hline Noise temperature & 10 \\
\hline Number of users per BS & $-10 \mathrm{~dB}$ \\
\hline Recommended I/N level & \\
\hline
\end{tabular}

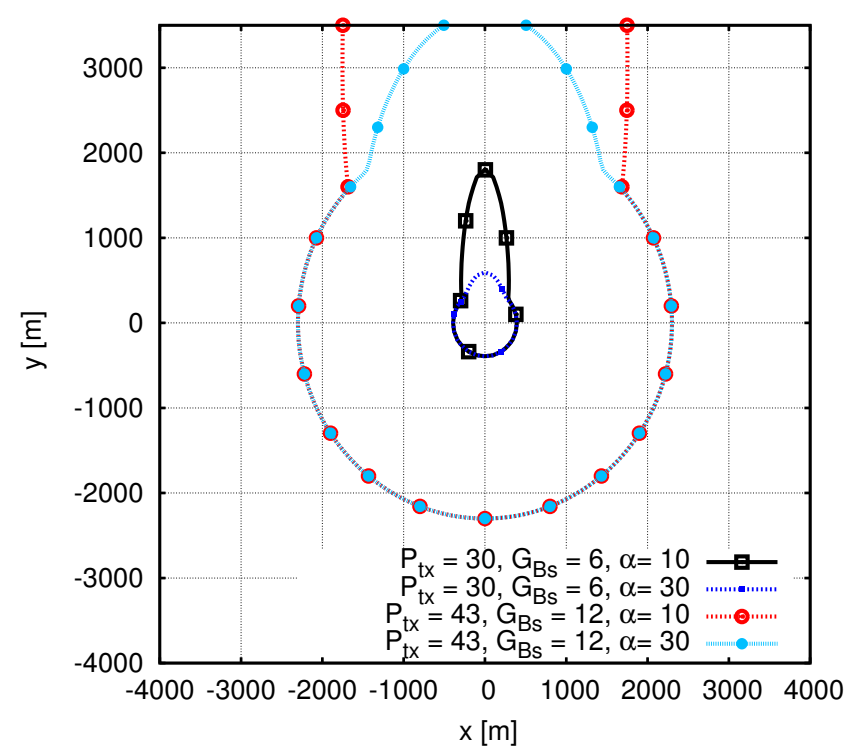

Fig. 4. $-10 \mathrm{~dB}$ region with a single interferer

FSS elevation angle $\alpha$ equal to $10^{\circ}$ and $30^{\circ}$. As expected, the $-10 \mathrm{~dB}$ area extension is proportional to the BS power. Moreover, the interference depends on the FSS elevation angle, in particular the higher the $\alpha$ the lower the interference at the FSS.

\section{B. Multiple omnidirectional BSs}

In the next simulations, we evaluate the interference at the FSS when more omnidirectional pico BSs are deployed around the FSS. In the first simulation, we consider the worst case scenario when one BS within every tier of BSs is placed in the direction of the maximum FSS antenna gain, i.e., $\vartheta=0^{\circ}$, and the others are placed accordingly with a fixed intersite distance $d_{i}$. Considering the expected cell coverage in next generation cellular networks [5] we assume $d_{i}=500 \mathrm{~m}$. Fig.

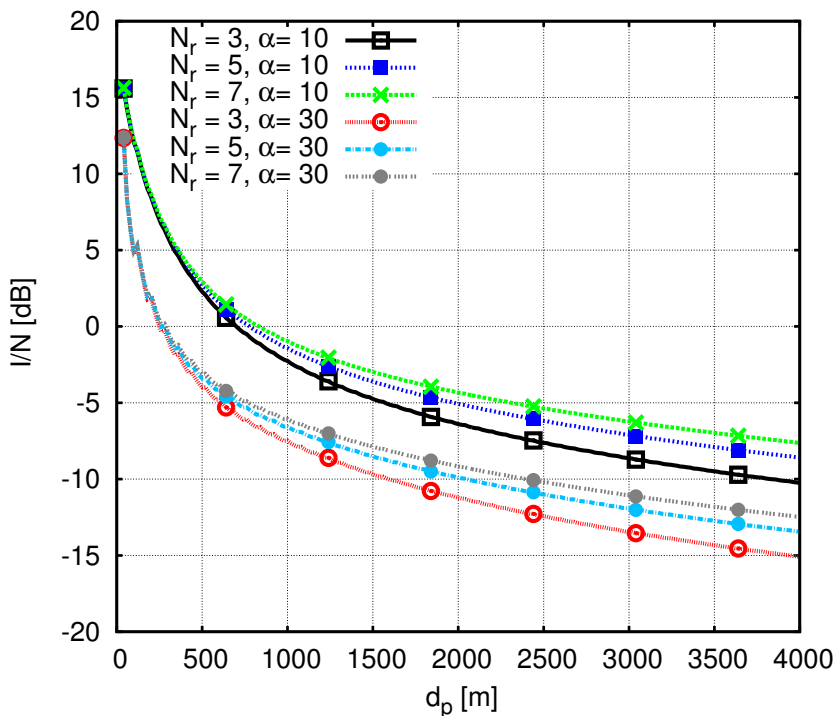

Fig. 5. Aggregate interference in the omnidirectional worst-case scenario

5 shows the $\mathrm{I} / \mathrm{N}$ obtained varying the protection distance and adopting three different values for the number of BS tiers, $N_{r}$, and two different elevation angles. We observe that, for $\alpha=10^{\circ}$, the interference at the FSS is very high and the $\mathrm{I} / \mathrm{N}$ level recommended by the standard is never achieved even for high $d_{p}$ values. Increasing the elevation angle to $30^{\circ}$ the recommended value is reached for $d_{p}=2000 \mathrm{~m}$. As expected, considering more BSs circles the interference at the FSS increases but the effects of the more distant BSs circles become negligible due to the high pathloss. Then, in the next evaluations, we restrict the value of $N_{r}$ to 3 or 5 .

Fig. 6 shows the cumulative distribution function (CDF) of $\mathrm{I} / \mathrm{N}$ when the $\mathrm{BSs}$ are deployed randomly over the circles around the FSS. We consider five tiers of BSs and two fixed protection distances for each value of the elevation angle. The intersite distance between the BSs is fixed as in the previous simulation. The results are obtained via Monte Carlo simulation with several runs per configuration where in each run the BS positions are changed. Firstly, we note that since the radiation pattern shape depends on $\alpha$, the higher the FSS elevation angle the lower the interference generated to the FSS and so the I/N level. In general, for the $d_{p}$ values considered the interference requirement is never met.

\section{Multiple directional BSs}

In Figs. 7, 8, and 9 we evaluate the interference at the FSS exploiting an analog beamforming scheme at the BSs that maximizes the user capacity. We consider a random scheduling pattern of the users. Fig. 7 shows the $\mathrm{I} / \mathrm{N}$ obtained in the worst-case scenario by varying the protection distance and considering different numbers of antennas at the transmitter $N_{a}$, and different values of $\alpha$. The number $N_{r}$ of tiers of BSs around the FSS is fixed to three and the results have been obtained, as in the previous case, by Monte Carlo evaluations over different channel realizations and user selections. When 


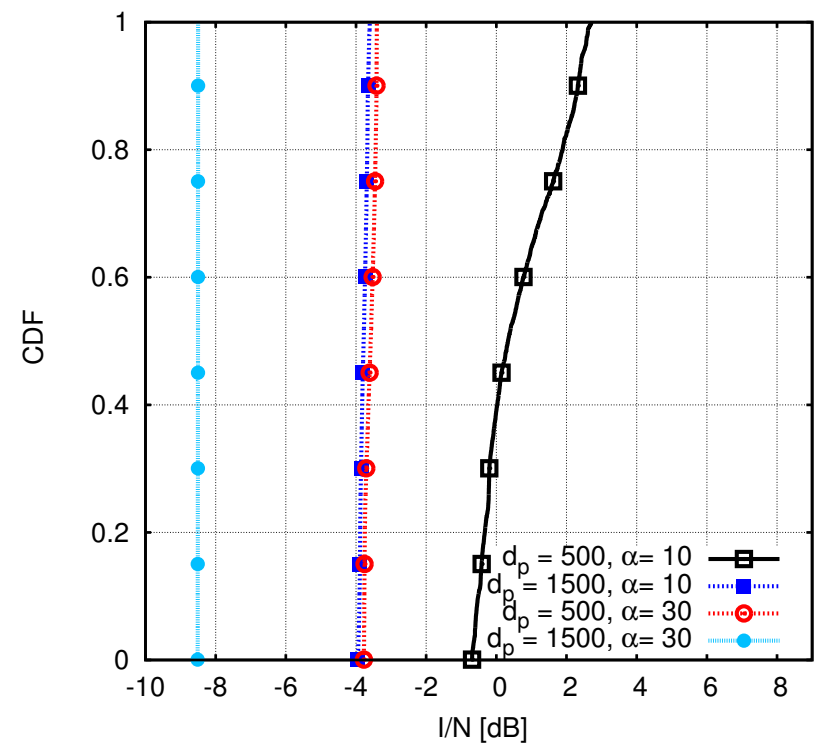

Fig. 6. Aggregate interference CDF with omnidirectional BSs

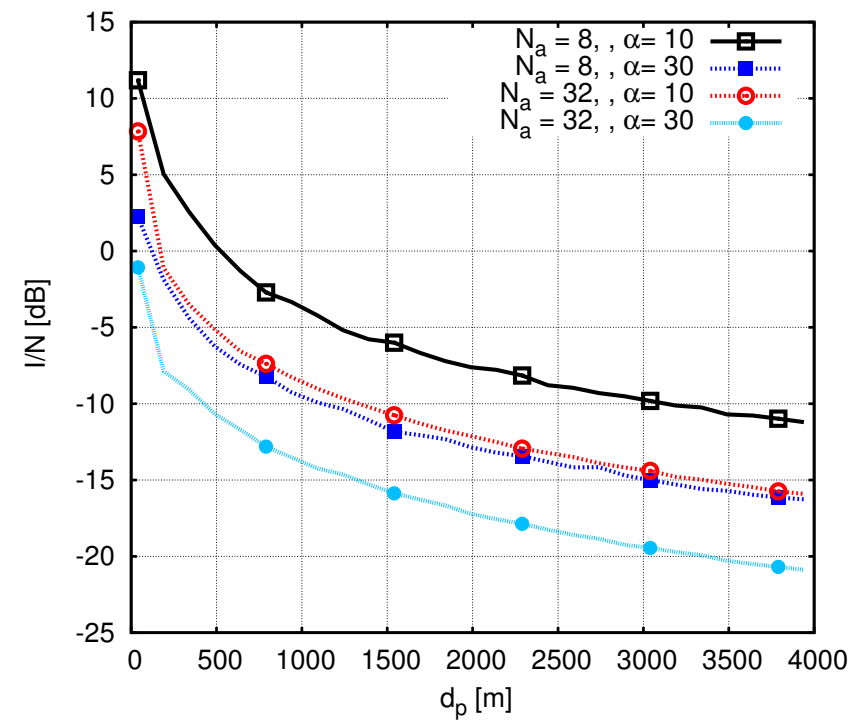

Fig. 7. Aggregate interference in the beamforming worst-case scenario

the number of antennas is increased, the directional beams become narrower and the interference due to the side lobes decreases. Also, the interference towards the FSS decreases with the number of antennas at the BSs. Besides, we note that the interference due to a small elevation angle can be compensated by using a larger antenna array. In general, beamforming schemes can reach a considerable improvement in comparison with the omnidirectional BSs case.

Fig. 8 shows the $\mathrm{CDF}$ of $\mathrm{I} / \mathrm{N}$ when the number of antennas is set to 16 and the BSs are randomly placed around the FSS over three tiers. Using this configuration, we notice that it is possible to satisfy the standard recommendation constraints adopting a protection distance of $1500 \mathrm{~m}$ for an elevation angle equal to

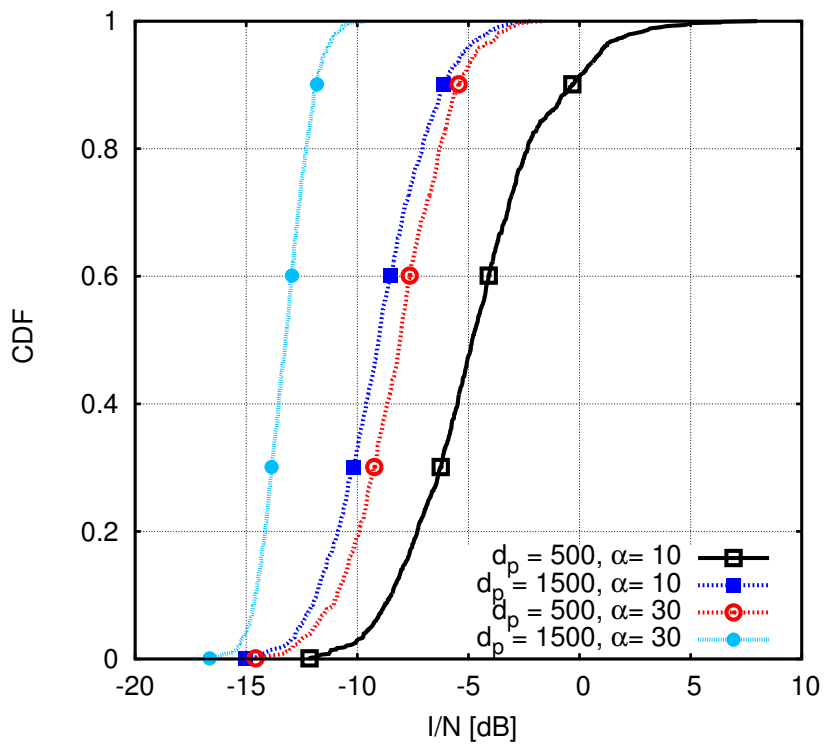

Fig. 8. Aggregate interference CDF considering BSs with $N_{a}=16$

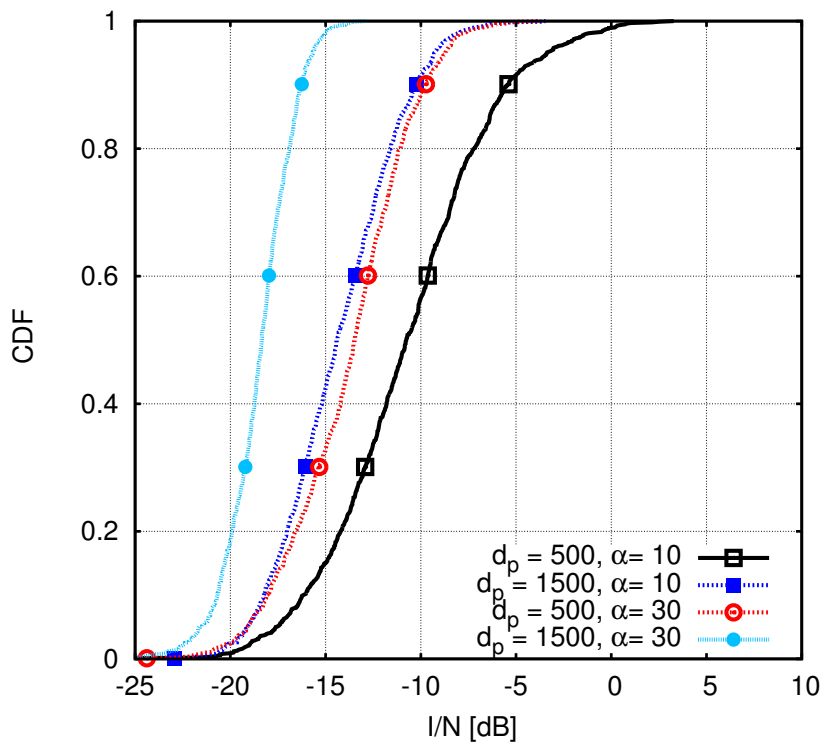

Fig. 9. Aggregate interference CDF considering BSs with $N_{a}=64$

$30^{\circ}$. We emphasize that user scheduling and beam selection are completely unaware of the interference at the FSS since no communication is assumed between the devices. The results of a similar evaluation are reported in Fig. 9 increasing the number of antennas at the BSs to 64 . We note that the required protection distance with $\alpha=30^{\circ}$ is reduced to $500 \mathrm{~m}$ and the regulatory requirement can be satisfied also for $\alpha=10^{\circ}$ with $d_{p}=1500 \mathrm{~m}$.

Fig. 10 shows the impact of the BSs intersite distance on the interference at the FSS. In this evaluation we set $N_{a}=16$, $d_{p}=1500, N_{r}=3$ and $\alpha=30$. As expected, decreasing the intersite distance, the BS density around the FSS increases, thus generating higher interference at the FSS. The BSs density 


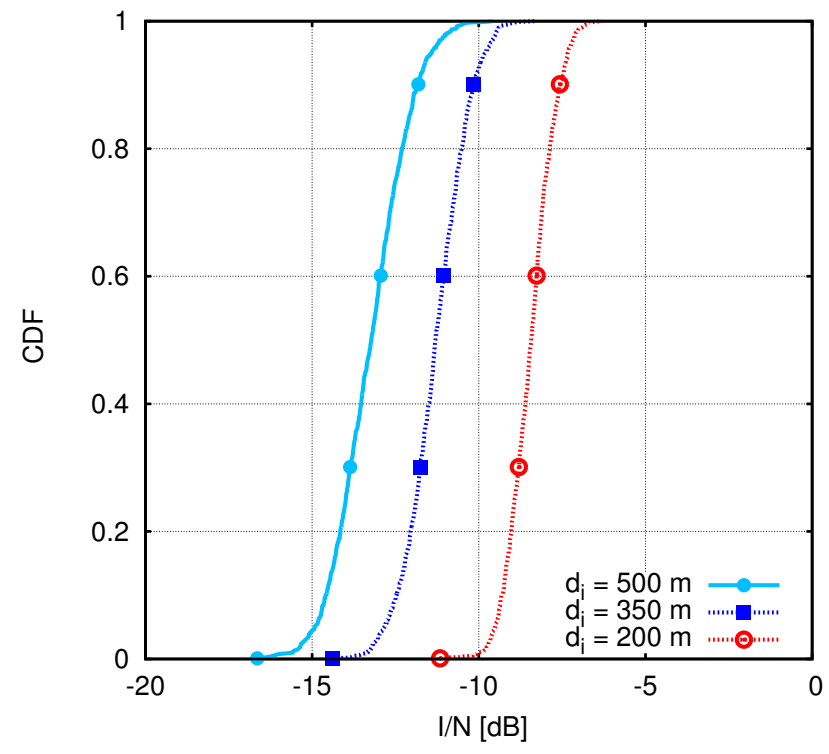

Fig. 10. Aggregate interference CDF considering different BSs intersite distances

is then another key parameter to design the network in order to preserve the FSS functionalities.

\section{CONClusions And Future Work}

In this paper, we investigated the coexistence feasibility of FSSs and cellular BSs in a mmWave scenario. We evaluated the $\mathrm{I} / \mathrm{N}$ at the FSS considering standard parameters and different BS deployments and configurations. In particular, we studied single and aggregate interference scenarios with a random multi-tier distribution of the BSs. Moreover, we analyzed the impact of the introduction of an RF beamforming scheme at the transmitters with the assumption of no interaction among FSS and BSs. From the results obtained, it appears that exploiting the characteristics of the mmWave scenario, such as high pathloss and large antenna arrays, the coexistence of FSSs and BSs in the same area is possible. Moreover, parameters such as the BS density, the protection distance and the FSS elevation angle became crucial in the network deployment to guarantee the FSS functionalities.

As future work, we intend to develop possible distributed cooperative algorithms of beamforming and scheduling among the BSs to mitigate the interference at the FSS. Finally, more complex scenarios such as heterogeneous networks and more realistic BSs deployments could be considered.

\section{ACKNOWLEDGMENT}

We thank Profs. Barry Evans and Klaus Moessner from 5G Innovation Centre for valuable discussion and inputs.

\section{REFERENCES}

[1] Cisco, Cisco Visual Networking Index: Global Mobile Data Traffic Forecast Update, 2013-2018, Feb. 2014, White Paper, pp. 1-40.
[2] T. S. Rappaport, S. Sun, R. Mayzus, H. Zhao, Y. Azar, K. Wang, G. N Wong, J. K. Schulz, M. Samimi, F. Gutierrez, "Millimeter wave mobile communications for 5G cellular: it will work!," IEEE Access, vol. 1, pp. 335-349, 2013.

[3] W. Roh, J. Y. Seol, J. Park, B. Lee, J. Lee, Y. Kim, J. Cho, K. Cheun, F. Aryanfar, "Millimeter-wave beamforming as an enabling technology for $5 \mathrm{G}$ cellular communications: theoretical feasibility and prototype results," IEEE Comm. Mag., pp. 106-113, Feb. 2014.

[4] T. Kim, J. Park, J. Seol, S. Jeong, J. Cho, W. Roh, "Tens of Gbps support with mmWave beamforming systems for next generation communications," IEEE GLOBECOM, pp. 3685-3690, 9-13 Dec. 2013.

[5] S. Rangan, T. S. Rappaport, E. Erkip, "Millimeter wave cellular wireless networks: potentials and challenges," Proc. of the IEEE, vol. 102, no. 3, pp. 366-385, March 2014.

[6] M. R. Akdeniz, Y. Liu, M. K. Samimi, S. Sun, S. Rangan, T. S. Rappaport, E. Erkip, "Millimeter wave channel modeling and cellular capacity evaluation," arXiv preprint arXiv: 1312.4921, 2013.

[7] T. Bai, V. Desai, R. W. Heath, "Millimeter wave cellular channel models for system evaluation," IEEE ICNC 2014, pp. 178-182, 3-6 Feb. 2014.

[8] S. Hur, Y. J. Cho, T. Kim, J. Park, A. Molisch, K. Haneda and M. Peter "Wideband Spatial Channel Model in an Urban Cellular Environments At $28 \mathrm{GHz}$," Proc. EuCAP 2015, April 2015.

[9] Metis project, "Intermediate description of the spectrum needs and usage principles," D5.1 Deliverable, Aug. 2013.

[10] CoRaSat project, "Service and market requirememts," D.2.1 Deliverable, Oct. 2013.

[11] ITU-R M.2109, "Sharing studies between IMT advanced systems and geostationary satellite networks in the fixed-satellite service in the 34004200 and 4500-4800 MHz frequency bands," 2007.

[12] ITU-R 4-5-6-7/TEMP/139-E, "Sharing and compatibility between IMT system and fixed-satellite service network in $5850-6425 \mathrm{MHz}$ frequency range," Feb. 2007.

[13] S. K. Sharma, S. Chatzinotas, B. Ottersten, "Satellite cognitive communications: Interference modeling and techniques selection," Proc. SPSC, pp. 111-118, 5-7 Sept. 2012.

[14] J. Lim, H. Jo, H. Yoon, J. Yook, "Interference mitigation technique for the sharing between IMT-advanced and fixed satellite service," Journ. of Comm. and Net., vol. 9, no. 2, pp. 159-166, June 2007.

[15] D. Oh, S. Lee, D. Ahn, S. Kim, "A Study on the Separation Distance for Frequency Sharing between GSO Network and Terrestrial Network in Ka Band,” Proc. IEEE VTC Spring, pp. 2967-2971, 11-14 May 2008.

[16] S. K. Sharma, S. Chatzinotas, B. Ottersten, "Transmit beamforming for spectral coexistence of satellite and terrestrial networks," Proc. IEEE CROWNCOM, pp. 275-281, 8-10 July 2013.

[17] F. Guidolin, M. Nekovee, L. Badia, M. Zorzi, "A Cooperative Scheduling Algorithm for the Coexistence of Fixed Satellite Services and 5G Cellular Network," Proc. IEEE ICC 2015.

[18] ITU-R P.452-15, "Prediction procedure for the evaluation of interference between stations on the surface of the Earth at frequencies above about $0.1 \mathrm{GHz}$, , Sept. 2013.

[19] ITU-R S.465, "Reference radiation pattern for earth station antennas in the fixed-satellite service for use in coordination and interference assessment in the frequency range from 2 to $31 \mathrm{GHz}$," Jan. 2010.

[20] A. Alkhateeb, O. El Ayach, G. Leus, R. W. Heath, "Hybrid precoding for millimeter wave cellular systems with partial channel knowledge," Proc. ITA Workshop, pp. 1-5, 10-15 Feb. 2013.

[21] ITU-R SF.558-2, "Maximum allowable values of interference from terrestrial radio links to systems in the fixed-satellite service employing 8-bit PCM encoded telephony and sharing the same frequency bands," July 1986.

[22] ITU-R Rec. S.1432, "Apportionment of the allowable error performance degradation to Fixed-Satellite Service (FSS) hypothetical reference digital paths arising from time invariant interference for system operating below $30 \mathrm{GHz}$, , Jan. 2000. 